\title{
Physician Use of Advance Care Planning Discussions in a Diverse Hospitalized Population
}

\author{
Sonali P. Kulkarni • Leah S. Karliner • \\ Andrew D. Auerbach · Eliseo J. Pérez-Stable
}

Published online: 18 July 2010

(c) The Author(s) 2010. This article is published with open access at Springerlink.com

\begin{abstract}
Two decades after the Patient Self Determination Act it is unknown how often physicians have advance care planning (ACP) discussions with hospitalized patients. The objective of this study is to investigate use of ACP discussions in a multi-ethnic, multi-lingual hospitalized population. Cross-sectional communication study of hospitalized patients. The Participants are 369 patients at one urban county hospital and one academic medical center. Interventions are not applicable. Participants were asked at baseline and a post-discharge interview whether hospital physicians had discussed either (a) what type of treatment they would want if they could not make decisions for themselves or (b) whether they would want cardiopulmonary resuscitation if needed. We compared patient characteristics for those who did and did not have an ACP discussion. Only $151(41 \%)$ participants reported an ACP discussion. Rates of ACP were low across ethnic, language, education and age groups. In a multivariate model, scoring higher on a co-morbidity scale was associated with higher odds of reporting having had an ACP discussion during hospitalization; this finding remained after adjusting for
\end{abstract}

\section{S. P. Kulkarni}

Robert Wood Johnson Clinical Scholars Program, Department

Medicine, University of California, Los Angeles, CA, USA

\section{S. Karliner · E. J. Pérez-Stable ( $\square)$}

Medical Effectiveness Research Center for Diverse Populations, Division of General Internal Medicine, Department of Medicine, University of California, 0320, 400 Parnassus Avenue,

San Francisco, CA 94143-0320, USA

e-mail: eliseops@medicine.ucsf.edu

\section{A. D. Auerbach}

Division of Hospital Medicine, Department of Medicine,

University of California, San Francisco, CA, USA time period and site of data collection. Multiethnic, multilingual hospitalized patients reported low rates of ACP discussions with their physicians regardless of ethnicity, English proficiency, education level or age.

Keywords Care discussion - Diverse populations . Hospital admission · Patient care planning

\section{Background}

The Patient Self Determination Act was passed as a measure to guarantee patient autonomy and involvement in medical decision making, particularly the right of patients to use advance directives to provide instructions for medical care should they become incapacitated [1]. Two decades later, there is evidence that a majority of patients have not participated in effective advance care planning either through completion of an advance directive or by having a meaningful discussion with their physician about their end of life care preferences such as desire for life sustaining treatments [2].

The use of advance care planning discussions with racial and ethnic minority populations, particularly those with limited English proficiency, has not been well studied in the literature despite research supporting the role that race, ethnicity, and culture play in end of life care planning [3]. Furthermore, Medicare expenditures were found to be $57 \%$ higher for Latino compared to White patients in the last six months of life [4]. Because ethnic minorities have been shown to be both less likely than Whites to have completed advance directives and less knowledgeable about the existence of advance directives [3], hospitalization may offer an opportunity for physicians to initiate advance care planning discussions with diverse patient populations. 
Given proposed national legislation to provide reimbursement for physician initiated advance care planning discussions, studies describing the use of these discussions in diverse populations are needed. We set out to investigate the prevalence of advance care planning discussions in a multi-ethnic, multi-lingual sample from two urban hospitals and examine patient factors that may be associated with such discussions.

\section{Methods}

\section{Study Population}

This cross-sectional study was part of a larger communication study focused on patients with limited English proficiency (LEP) which recruited a cohort of hospitalized patients from the medical and surgical wards of two large urban hospitals between January 2005 and May 2008. In the interest of maximizing ethnic and language diversity, we recruited participants primarily from a safety net hospital with a largely Latino and African American population, and supplemented our sample with participants from an academic medical center with a larger Asian population to specifically capture Chinese speakers. We attempted to recruit all eligible LEP patients admitted during the study period [5], and achieved a collaboration rate for LEP patients of $71 \%$.

In accordance with Federal and California state regulations, the medical centers in our study require their admitting personnel (non-physicians) to ask patients whether they have a pre-existing advanced directive or a similar physician order for life sustaining treatment; if they do not, they are given information related to their right to have an advanced healthcare directive. There is no formal Federal, state, or hospital requirement that physicians initiate a discussion with patients regarding their desires for treatment should they become incapacitated.

\section{Procedures}

Participants were interviewed both at a baseline inpatient in-person interview and at a two week post-discharge telephone interview by a bilingual research assistant in Spanish, Chinese, or English according to patient preference. The overall follow-up rate at the 2 week post-discharge interview was $84 \%$. This analysis includes the 369 patients for whom we had complete baseline and chart data.

\section{Measurements}

Participants self reported their gender, age, educational attainment, race or ethnicity, and English proficiency. They were categorized as having LEP if they answered the question "How well do you speak English?" either with "not at all" or "not well", or if they answered "well", but stated a preference to receive their medical care in another language [5]. Participants' comorbidity score was obtained by counting the number of comorbidities present using an adaptation of the validated Self-Administered Comorbidity Questionnaire [6]. Information regarding admitting service was obtained from chart review.

Participants were asked in both the baseline and postdischarge interview whether hospital physicians had discussed either (a) what type of treatment they would want if they could not make decisions for themselves, and/or (b) whether they would want cardiopulmonary resuscitation (CPR) if needed. Participants were categorized as having discussed advance care planning if the reply was "yes" to either question at either time-point.

\section{Statistical Analysis}

We assessed the bivariate association of advance care planning discussion with the following patient factors: age, gender, ethnicity, educational attainment, English proficiency, comorbidity, admitting service, recruitment site using chi-square and t-tests as appropriate. We performed logistic regression to identify predictors of advance care planning discussion. To attain the best subset of predictors, we used backward selection, entering all variables significant at the $P<0.2$ level, except for race/ethnicity, which was not entered into the model due to the highly significant relationship with English proficiency. For all analyses, we used SAS version 9.2 (SAS Institute Inc, Cary, NC).

\section{Results}

Among the 369 participants in our study, a majority (66\%) were less than 50 years old (mean age 44; range 18-88) and approximately half (49\%) were women. Our study population was racially and ethnically diverse with $63 \%$ Latinos, 16\% African Americans, 13\% Asian Americans, and $8 \%$ Whites. Sixty-three percent reported limited English proficiency and only $41 \%$ had completed a high school education or greater. A majority were admitted to a surgical service $(58 \%)$ and the average number of comorbidites was 2.1 (std.dev. 1.8; range 0-8). Among the participants with LEP, only two were born in the US; average length of time since immigrating to the US was 10.5 years (range $1-58$; sd \pm 9.5 ).

Only $151(41 \%)$ patients reported an advance care planning discussion with a physician during their hospitalization. Among those who answered questions about advanced care planning discussions in both the baseline 
Table 1 Report of advance care planning discussions in 369 hospitalized patients, San Francisco Bay Area, 2005-2008

\begin{tabular}{|c|c|c|c|}
\hline & $\begin{array}{l}\text { ACP discussion } \\
N=151(\%)\end{array}$ & $\begin{array}{l}\text { No ACP discussion } \\
N=218(\%)\end{array}$ & $P$-value \\
\hline \multicolumn{4}{|l|}{ Age } \\
\hline$<50$ & $101(41)$ & $143(59)$ & \multirow[t]{2}{*}{0.80} \\
\hline$\geq 50$ & $50(40)$ & $75(60)$ & \\
\hline \multicolumn{4}{|l|}{ Gender } \\
\hline Men & $72(38)$ & $118(62)$ & \multirow[t]{2}{*}{0.22} \\
\hline Women & $79(44)$ & $100(56)$ & \\
\hline \multicolumn{4}{|l|}{ Education } \\
\hline$<\mathrm{HS}$ & $82(38)$ & $135(62)$ & \multirow[t]{2}{*}{0.16} \\
\hline HS grad or more & $68(45)$ & $83(55)$ & \\
\hline \multicolumn{4}{|l|}{ Ethnicity } \\
\hline Latino & $85(37)$ & $147(63)$ & \multirow[t]{4}{*}{0.17} \\
\hline African American & $27(46)$ & $32(54)$ & \\
\hline Asian American & $23(49)$ & $24(51)$ & \\
\hline White & $16(52)$ & $15(48)$ & \\
\hline \multicolumn{4}{|l|}{ English proficiency } \\
\hline Limited English proficient & $88(38)$ & $144(62)$ & \multirow[t]{2}{*}{0.13} \\
\hline English proficient & $63(46)$ & $74(54)$ & \\
\hline \multicolumn{4}{|l|}{ Admitting service } \\
\hline Surgical or $\mathrm{OB} / \mathrm{GYN}$ & $93(43)$ & $121(57)$ & \multirow[t]{2}{*}{0.24} \\
\hline Medical & $58(37)$ & $97(63)$ & \\
\hline Comorbidity (mean \pm SD) & $2.4 \pm 1.8$ & $1.9 \pm 1.7$ & 0.004 \\
\hline
\end{tabular}

and follow-up interviews $(n=309)$ and who reported no discussion at baseline $(n=224), 22 \%$ reported at followup that they had a discussion. Of those who reported at baseline that they had a discussion $(n=85), 34 \%$ reported no discussion at follow-up.

Participants who reported an advanced care planning discussion did not significantly differ from those who reported no discussion by age, ethnicity, or education or gender (Table 1). Likewise, participants' English proficiency was not associated with report of advance care planning discussions; and, among LEP participants, length of time since immigrating to the US was also not associated with report of such discussions. Patients admitted to a surgical service were as likely to have such discussions as those admitted to a medical service. Participants who reported an advance care planning discussion were, however, more likely to have a higher average number of comorbidities $(2.4 \pm 1.8$ vs. $1.9 \pm 1.7, P=0.004)$. Patients recruited at the academic medical center were more likely to have an advance care planning discussion compared to those recruited at the safety net hospital (61 vs. $38 \%, P=0.002$ ). However, those at the academic medical center were also more than twice as likely to be older than 50 years old (73 vs. $28 \%, P<0.001$ ) and to have a higher average number of comorbidities $(3.1 \pm 1.7$ vs. $2.0 \pm 1.7, P<0.0001)$.

Backward selection removed education, gender, and LEP status and resulted in a model showing an association of comorbidity score with advance care planning discussion adjusted for time period and site of data collection. For each one-point increase in the comorbidity score, the odds of having an advanced care planning discussion increased $15 \%$ (OR 1.15; 1.02-1.30).

\section{Discussion}

Advance care planning is a complex process that necessitates discussion between patients, their loved ones, and their physicians. In our multi-ethnic, multi-lingual sample of hospitalized patients, only $41 \%$ of patients reported having an advance care planning discussion with a hospital physician. While this is improved from previous studies that documented use of advance care planning discussions among seriously ill hospitalized patients to be less than $25 \%,[7,8]$ the prevalence remains disturbingly low. In addition, a third of participants who reported having had ACP discussion when interviewed in the hospital did not recall having had that discussion when interviewed in the month following discharge. This may be in part because the participant's health had improved enough that the discussion was no longer salient; however, it may also be indicative of low-efficacy discussions.

We found that comorbidity score was significantly associated with increased use of advance care planning 
discussions, and this finding was robust to adjustment for time-period and recruitment site. Physicians have been recognized as being in the best position to initiate and guide advance care planning discussions [8, 9], and research indicates that patients with chronic illness are in particular need of advance care planning [2]. Therefore, it is not surprising, and is even encouraging, that clinical information such as a high comorbidity score is associated with a physician initiating such a discussion. We also found substantially higher use of ACP discussions at the academic medical center compared to the safety net institution; while this finding is at least in part related to differences in patient co-morbidity, it is also consistent with other research documenting variation in the use of ACP or code status discussions by hospital site and suggests the strong role of local culture in their use [10].

Racial and ethnic minorities have lower rates of documented advance directives compared to Whites [3], and incur higher healthcare cost due to higher rates of intensive treatment at the end of life [4]. While distrust and cultural specific attitudes are thought to play a role in making advance directives more problematic particularly among less acculturated adults [3], it is unclear if more intervention at the end of life is due to preference or to ineffective communication about the utility of these interventions or palliative options. [4] We hypothesized that cultural differences and language barriers may in some way contribute to decreased physician use of advance care planning discussions with their minority patients. In our study, however, we did not find an association between level of formal education, ethnicity, English proficiency or, for immigrant participants, length of time living in the US and patient report of advance care planning discussion. These findings suggest that in this hospitalized patient sample, these factors do not significantly influence patient reports of physician use of advance care planning discussions. It is possible that ACP discussions are only used or relevant in the care of patients when there are medical problems that indicate a high likelihood of significant clinical worsening. However, the sample size limits our ability to definitively state that among older, less educated LEP patients, there are no differences in ACP discussions.

Our results should be viewed in light of several limitations. While we did not observe differences by age, the overall young age of patients at the safety net hospital likely influenced physicians against the use of advance care planning discussions and contributed to lower rates of ACP discussion. Also, we did not have access to additional patient information, such as severity of acute illness, which may be a major factor in physician decision to discuss advance care planning during hospitalization, and has been used in prior studies [7]. Lastly, we relied solely on patient report of discussions, which may be subject to recall bias; however, patient participation in, understanding and recall of these discussions ultimately is the most important outcome.

\section{Conclusion}

In a multiethnic, multi-lingual sample of hospitalized patients, patients reported low rates of advance care planning discussions. This finding held true regardless of ethnicity, English proficiency, education level or age.

\section{IRB Approval}

This study was approved by the institutional review boards of both medical centers from which patients were recruited, including the University of California San Francisco IRB.

Acknowledgments This study was supported by grant no. 20061003 from the California Endowment and by grant no. P30AG15272 of the Resource Centers for Minority Aging Research program funded by the National Institute on Aging, the National Institute of Nursing Research, and the National Center on Minority Health and Health Disparities, National Institutes of Health. We thank Dana Nickleach for data analysis support.

Conflicts of interest The authors are not aware of any conflict of interest with regard to this study or manuscript.

Open Access This article is distributed under the terms of the Creative Commons Attribution Noncommercial License which permits any noncommercial use, distribution, and reproduction in any medium, provided the original author(s) and source are credited.

\section{References}

1. Patient Self-Determination Act. 42 USC 1395cc, 1396a. 1994.

2. Agency for Healthcare Research and Quality. Advance care planning: Preferences at the end of life. research in action, Issue 12. Available at: http://www.ahrq.gov/research/endliferia/endria. htm\#NeedMore. Accessed 30 June 2009.

3. Kwak J, Haley WE. Current research findings on end-of-life decision making among racially or ethnically diverse groups. Gerontologist. 2005;45:634-41.

4. Hanchate A, Kronman AC, Young-Xu Y, Ash AS, Emanuel E. Racial and ethnic differences in end-of-life costs: why do minorities cost more than whites? Arch Intern Med. 2009;169(5): 493-501.

5. Karliner LS, Napoles-Springer A, Schillinger D, BibbinsDomingo K, Perez-Stable E. Identification of limited English proficiency patients: does the U.S. census question measure up? J Gen Intern Med. 2008;23:1555-60.

6. Sangha O, Stucki G, Liang MH, Fossel AH, Katz JN. The selfadministered comorbidity questionnaire: a new method to assess comorbidity for clinical and health services research. Arthritis Rheum. 2003;49:156-63. 
7. Hofmann JC, Wenger NS, Davis RB, et al. Patient preferences for communication with physicians about end-of-life decisions. SUPPORT Investigators. Study to understand prognoses and preference for outcomes and risks of treatment. Ann Intern Med. 1997;127:1-12.

8. Mirza A. Cardiopulmonary resuscitation is not addressed in the admitting records for the majority of patients who undergo CPR in the hospital. Am J Hosp Palliat Care. 2005;22:20-5.
9. Emanuel LL, Danis M, Pearlman RA, et al. Advance care planning as a process: structuring discussions in practice. J Am Geriatr Soc. 1995;43:440-6.

10. Auerbach AD, Katz R, Pantilat SZ, et al. Factors associated with discussion of care plans and code status at the time of hospital admission: results from the multicenter hospitalist study. J Hosp Med. 2008;3:437-45. 